\title{
Diffusive persistence and the 'sign-time' distribution
}

\author{
T. J. Newman and Z. Toroczkai \\ Department of Physics, \\ Virginia Polytechnic Institute and State University, \\ Blacksburg VA 24061, \\ $U S A$
}

\begin{abstract}
We present a new method for extracting the persistence exponent $\theta$ for the diffusion equation, based on the distribution $P$ of 'sign-times'. With the aid of a numerically verified Ansatz for $P$ we derive an exact formula for $\theta$ in arbitrary spatial dimension $d$. Our results are in excellent agreement with previous numerical studies. Furthermore, our results indicate a qualitative change in $P$ above $d \simeq 36$, signalling the existence of a sharp change in the ergodic properties of the diffusion field.
\end{abstract}

PACS numbers: 05.40.+j, 82.20.-w

In the past few years there has been much interest in calculating the persistence properties for a wide range of simple model systems. Examples are the diffusion equation [1], the Ising model with Glauber dynamics [2] and its Potts model generalization [3], interface kinetics [4, phase ordering $\mid 5]$, and the voter model $[6]$. Perhaps the simplest and most generic system is the first - the diffusion equation. Naively one might expect that everything is known about such a classical system. However, one needs only reflect upon its intimate relation to the rich Burgers model of turbulence [7] (obtained via a simple non-linear transformation) to appreciate the potential complexity of diffusion physics.

This complexity was again uncovered by studies of diffusive persistence [1]. The persistence exponent $\theta$ for this case is defined as follows. Consider the deterministic diffusion equation evolving a random initial condition (usually created from an uncorrelated gaussian distribution). Then consider the probability $q(t)$ that the diffusion field at a given site has never changed sign. One finds numerically that this probability decays with time in a power-law fashion, with an exponent $\theta$, whose value is not a simple rational number. There is no analytic prediction for $\theta$ with the exception of the results from the 'independent interval approximation' (IIA) which are in good agreement with numerical work in spatial dimension $d=1$, but fare less well in higher dimensions [1]. It is easy to find applications for diffusive persistence due to the ubiquitous presence of diffusion physics. Examples include: survival of reactants in reaction kinetics, and more general survival probabilities in systems with a field slaved to a diffusion process.

In this Letter we shall present an exact analytic form for $\theta$. The key to our derivation is that one may obtain $\theta$ by studying a more general quantity; namely the distribution $P$ of 'sign-times' $\tau(t)$ (to be defined below), which has also been recently introduced by Dornic and Godrèche [8]. This distribution may be shown to have an exact scaling form for all $t$ : $P d \tau=f(\tau / t)(d \tau / t)$. Numerically the scaling function $f$ is found to be extremely simple. Using this form of $f$ as an Ansatz, allows an exact determination of $\theta$. Our prediction deviates by $3 \%$ from the numerically determined value in $d=1$, but lies well within the error bars of the simulation results in $d=2$ and $d=3$. We shall discuss the reasons for the slight $d=1$ discrepancy toward the end of the Letter. We also find that the qualitative nature of $P$ goes through a sharp transition at $d_{c} \simeq 36$.

We consider the evolution of a scalar field $\phi(\mathbf{r}, t)$ which satisfies

$$
\partial_{t} \phi=D \nabla^{2} \phi
$$

with initial condition $\phi(\mathbf{r}, 0)=\psi(\mathbf{r})$, where the field $\psi$ is an uncorrelated random variable described by a gaussian distribution

$$
R[\psi] \sim \exp \left[-(1 / 2 \Delta) \int d^{d} r \psi(\mathbf{r})^{2}\right] .
$$

The solution of Eq.(1) has the form

$$
\phi(\mathbf{r}, t)=\int d^{d} r^{\prime} g\left(\mathbf{r}-\mathbf{r}^{\prime}, t\right) \psi\left(\mathbf{r}^{\prime}\right),
$$

where $g(\mathbf{r}, t)=(4 \pi D t)^{-d / 2} \exp \left(-r^{2} / 4 D t\right)$ is the heat kernel.

The most obvious way to approach the persistence problem is to directly calculate the probability that the field at a given site ( $\mathbf{r}=\mathbf{0}$ say) has never changed sign. This amounts to the evaluation of

$$
q(t)=\left\langle\prod_{t^{\prime}=0}^{t} \theta\left(\phi\left(\mathbf{0}, t^{\prime}\right)\right)\right\rangle_{R},
$$

where $\theta(z)$ is the Heaviside step function [9]. Apart from the IIA (which is difficult to systematically improve) there does not seem to be any possibility of calculating this average.

Let us now focus our attention on 'sign-times' $\tau(t)$ defined as 


$$
\tau(t)=\int_{0}^{t} d t^{\prime} \theta\left(\phi\left(\mathbf{0}, t^{\prime}\right)\right),
$$

so that $\tau(t) / t$ is the fraction of time $t$ in which the field at $\mathbf{r}=\mathbf{0}$ was positive. Note that this is a much easier object to handle than $q(t)$ since we do not require the positivity of $\phi$ for a continuous succession of times. We simply follow the evolution of $\phi$ and record how often it is positive. Now, the sign-time $\tau(t)$ is actually a functional of the random variable $\psi$, and as such is described by some probability distribution $P[\tau, t]$ (see also Ref. [8]). We can immediately list a few properties of $P$. First, it is defined in the domain $[0, t]$. Second, it is symmetric about $\tau=t / 2$ (since it does not matter whether we study how often $\phi$ is positive, or how often $\phi$ is negative). Third, and most important, the behaviour of $P$ near $\tau=0$ or $t$ directly furnishes us with the exponent $\theta$. This is clear, since the probability for $\tau$ to be in the vicinity of either 0 or $t$ is nothing more than $q(t)$ defined above.

We refer the reader to Appendix A in which we prove an important fourth property of $P$. Namely, that it assumes the scaling form $P[\tau, t] d \tau=f(\tau / t)(d \tau / t)$ for any non-zero time $t$. [Note that $P$ does not depend on the model parameters $D$ and $\Delta$.] Given the symmetry of $P$ about $\tau=t / 2$, we may rewrite the scaling form as

$$
P[\tau, t] d \tau=g(x(1-x)) d x,
$$

where $x=\tau / t$ and we have assumed $f$ to be analytic around $x=1 / 2$. The third property listed above imposes that $g(y) \sim y^{\theta-1}$ for $y \ll 1$, where $y=x(1-x)$. So we have reduced the persistence problem to that of calculating the tail of the 'sign-time' distribution. This is still a formidable task, as it involves calculating arbitrarily high moments of the distribution (see Appendix A). As an alternative strategy we make an Ansatz. Namely, that the small-y form for $g$ actually holds for all $y$ in the available range $y \in[0,1 / 2]$. This very simple Ansatz was both suggested and confirmed to us by the results of our numerical work, which we now briefly describe. (Note also that this Ansatz appears naturally within the framework of the IIA [8].)

Following previous work [1], we model the diffusion equation by a discrete space-time process

$$
\phi_{i}(t+1)=\phi_{i}(t)+a \sum_{j}\left[\phi_{j}(t)-\phi_{i}(t)\right]
$$

where the sum is over nearest neighbours of $i$ on a $d$ dimensional hypercubic lattice. The parameter $a$ is chosen to be $1 /(2 d)$. The initial value of each $\phi_{i}(0)$ is drawn from a gaussian distribution. Simulations are performed on large lattices $\left(N \sim 2^{20}\right.$ sites) for times up to $t \sim 2^{10}$ with several independent runs. We measure two quantities during the simulation. First, we record the number $n(t)$ of those sites at which the field has never changed sign. The ratio of $n / N \sim t^{-\theta}$ (due to the self-averaging of the system). We also record the sign-time for each site and thus construct the histograms for $P[\tau, t]$. Our results for the former quantity are in agreement with those of Ref. [1]. In Figs. 1 and 2 we show the histograms for $d=1$ and $d=2$ respectively. The results are plotted on a log-log scale, since the data and the Ansatz are indistinguishable on a linear scale. One sees that the scaling function $g$ indeed varies as a simple power law in $y$ over the range $y \in[0,1 / 2]$. The only region in which there is a deviation from this power-law behaviour is near $y=0$. This is purely a (time)-lattice effect, since the histogram has a finite number of bins given by the number of timesteps. As $t$ increases, more and more bins are supplied near $x=0$ (where the function is integrably singular) and the expected power-law behaviour stretches closer and closer to the origin. We stress that the power-law behaviour near $x=0$ is guaranteed by the existence of the persistence exponent $\theta$. Our a priori knowledge of $g$ is limited in precisely that region of $x(\in[\varepsilon, 1 / 2])$ where the numerical results show a clear single power-law behaviour. We have added straight-lines on the plots, which have slopes of $\theta-1$, with values of $\theta$ taken from Eq.(11) below.

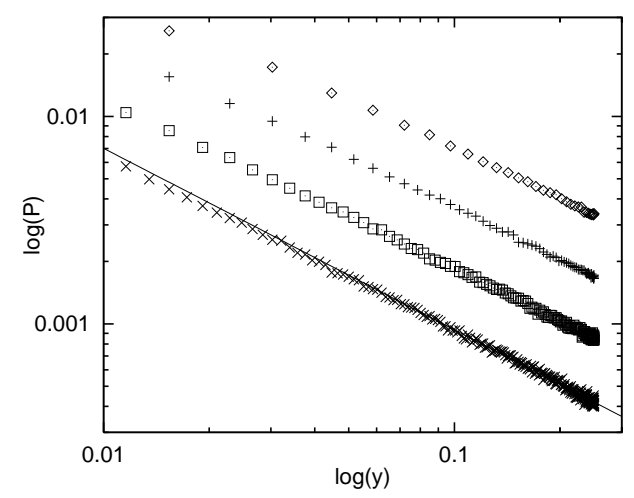

FIG. 1. A log-log plot of the sign-time distribution $P$ against $y$ for $d=1$. The data are taken at times $t=2^{n}$ with $n=6,7,8,9$ from top to bottom. The straight line is a power law with slope $\theta-1, \theta=0.1253$.

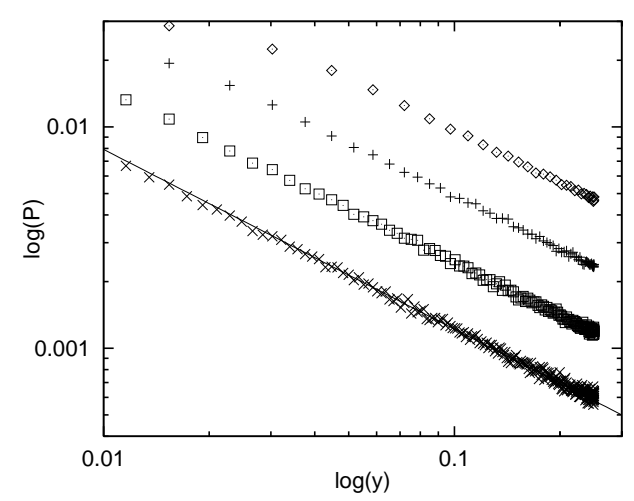


FIG. 2. As above, with $d=2$ and $\theta=0.1879$.

We are now in a position to calculate $\theta$. The numerical results clearly support $P[\tau, t]=(c / t)[x(1-x)]^{\theta-1}$. This distribution contains only two parameters: an amplitude $c$ (to be set by normalization), and the exponent $\theta$. The latter can in principle be set by the calculation of any even moment of $P$ (since the odd moments contain no new information, as the odd cumulants are zero). The simplest to consider is obviously the second moment $\mu_{2}$. (Unfortunately it is extremely difficult to calculate any even moments above the second.) The integrals over $P$ are simply given in terms of the Beta function 10. From the normalization one fixes $1 / c=B(\theta, \theta)$. The calculation of the second moment yields

$$
\mu_{2}=\frac{B(\theta+2, \theta)}{B(\theta, \theta)}=\frac{(1+\theta)}{2(1+2 \theta)} .
$$

We may now independently calculate $\mu_{2}$ from the original definition of the sign-times. Explicitly we have:

$$
\mu_{2}=\left\langle(\tau(t) / t)^{2}\right\rangle_{R}=\int_{0}^{1} d a_{1} \int_{0}^{1} d a_{2} C_{2}\left(a_{1} t, a_{2} t\right),
$$

where $C_{2}=\left\langle\theta\left(\phi\left(\mathbf{0}, a_{1} t\right)\right) \theta\left(\phi\left(\mathbf{0}, a_{2} t\right)\right)\right\rangle_{R}$. This latter quantity may be calculated exactly to give (see Appendix for a hint)

$$
C_{2}=\frac{1}{4}+\frac{1}{2 \pi} \sin ^{-1}\left[\left(\frac{2 a_{1}^{1 / 2} a_{2}^{1 / 2}}{\left(a_{1}+a_{2}\right)}\right)^{d / 2}\right] .
$$

The integrals in (9) are easily performed, leaving one with the expression

$$
\mu_{2}=\frac{1}{4}(2-\beta) \quad \Rightarrow \quad \theta=\frac{\beta}{2(1-\beta)},
$$

where

$$
\beta(d)=\frac{d}{2 \pi} \int_{0}^{1} d a \frac{(1-a)}{(1+a)}\left[\left(\frac{1+a}{2 a^{1 / 2}}\right)^{d}-1\right]^{-1 / 2} .
$$

This integral may be performed explicitly in one and two dimensions, with the results:

$$
\begin{gathered}
\beta(1)=\frac{1}{2^{3 / 2} \pi}[\psi(11 / 8)+\psi(9 / 8)-\psi(7 / 8)-\psi(5 / 8)] \\
=8 \sqrt{2} / 3 \pi-1=0.20042 \cdots,
\end{gathered}
$$

and

$$
\begin{aligned}
\beta(2)=\frac{1}{\pi}[\psi(5 / 4) & -\psi(3 / 4)] \\
& =4 / \pi-1=0.27323 \cdots,
\end{aligned}
$$

where $\psi(z)$ is the digamma function [10], showing that the persistence exponent is not a simple rational number, but is transcendental. We refer the reader to Table 1 where values of $\theta(d)$ are listed, along with the numerical and IIA estimates of Ref. [1].

It is of interest to calculate the large- $d$ form for $\theta$. Then one finds:

$$
\theta(d)=\frac{\pi}{4 I}\left(\frac{d}{2}\right)^{1 / 2}+O(1)
$$

where

$$
I=\int_{0}^{\infty} d w \frac{[\log (1+w)]^{1 / 2}}{w^{1 / 2}(1+w)}=3.0005 \cdots,
$$

giving a value of $\theta(d) \sim(0.1850 \cdots) \sqrt{d}$. This is to be compared to the result from the IIA which gives $\theta_{\text {IIA }}(d) \sim(0.1454 \cdots) \sqrt{d}$. As can be seen from this large$d$ limit, and also from Table I, the IIA consistently underestimates the value of $\theta$.

The fact that $\theta$ passes through unity is very interesting as it has a direct consequence for the sign-time distribution $P$. For $\theta<1, P$ has integrably divergent tails at $\tau(t) \rightarrow 0$ and $\tau(t) \rightarrow t$. Also, the mean of the $P$ (which is at $\tau=t / 2$ ) is the least likely value of $\tau$. One can understand this by considering a given point being located in the centre of a very large positive domain. A long time must pass before a negative domain sweeps through, thus halting the sign-time clock. However, if $\theta>1$, the distribution becomes convex, and the tails go to zero at the end-points of the range $[0, t]$. Thus the mean value $\tau=t / 2$ is in this case the most likely value of $\tau$. The dynamic mixing of positive and negative domains is in this case very efficient. (We shall return to this point in our conclusions.) It is therefore important to know at what dimension $\theta$ passes through unity. This is equivalent to insisting that $\beta\left(d_{c}\right)=2 / 3$. Numerical evaluation of the integral in Eq.(12) using Mathematica, yields the value $d_{c}=35.967 \cdots$. The fact that this enormous dimension plays a physical role in diffusion physics is extraordinary at first sight: such is the complexity of diffusive persistence.

\begin{tabular}{|c||c|c|c|}
\hline$d$ & $\theta$ & $\theta_{\text {IIA }}$ & $\theta_{\text {sim }}$ \\
\hline 1 & $0.1253 \cdots$ & $0.1203 \cdots$ & $0.1207(5)$ \\
\hline 2 & $0.1879 \cdots$ & $0.1862 \cdots$ & $0.1875(10)$ \\
\hline 3 & $0.2390 \cdots$ & $0.2358 \cdots$ & $0.2380(15)$ \\
\hline$\gg 1$ & $0.1850 \cdots \sqrt{d}$ & $0.1454 \cdots \sqrt{d}$ & - \\
\hline
\end{tabular}

Table 1: The calculated value of $\theta$ from Eqs.(11) and (12), along with the IIA and simulation estimates from Ref. [1].

Before concluding, we wish to make some comments regarding the role of the space-time lattice. It is quite 
possible to set up a calculation of the persistence exponent, or the sign-time distribution, with the discrete algorithm (7) as a starting point. This formulation has the advantage that the direct calculation of $q(t)$, as defined by Eq.(A), is well-defined. [In the continuum a microscopic time cut-off must be introduced to make sense of the time slices.] We have also pursued the discrete formulation in an attempt to calculate $P[\tau, t]$. As with the continuum case, it is extremely difficult to calculate any even moment above the second. However, we find the interesting result that the second moment contains strong corrections to scaling; i.e. $\left\langle\tau(t)^{2}\right\rangle \sim t^{2}+O(t)$. In the continuum, each moment has a clean scaling $\left\langle\tau(t)^{n}\right\rangle \sim t^{n}$ for any non-zero time. The implication of this result, is that one should not expect true scaling from numerical work (based on (7)) until very late times. This is especially true in low dimensions where the lattice Laplacian is much weaker than its continuum counterpart. This effect is already apparent in the histograms shown in Figs. 1 and 2. One sees that the tail (for small $y$ ) bends away from its power-law form due to the discrete sampling on a finite time grid. As more time steps are used, this deviation from scaling is pushed to smaller values of $y$. It should be stressed that the direct measurement of persistence from the ratio $n(t) / N$ is less reliable since the measurement is equivalent to sampling $P$ at $y=0-$ precisely in the region most affected by finite time-step effects. It is for these reasons that we believe the $d=1$ measurement of $\theta$, as given in Ref. [1], to be the least solid. In principle one can avoid these finite time-grid effects by sampling data from the exact solution of the diffusion equation, as given in (3), where $t$ is a real, continuous quantity. Such a numerical study would require orders of magnitude more computer time than the previous studies, but could potentially give a definitive answer for the case $d=1$.

In conclusion we have provided an exact form for the diffusive persistence exponent $\theta$, with the aid of an Ansatz based on numerical observations of the sign-time distribution $P$. Naturally, this result is in no way rigorous. We consider a proof of our Ansatz to be a reachable goal, although such a proof involves very technical manipulations in $n$-dimensional geometry 11,12 . We have also demonstrated the wider significance of the persistence exponent in parameterizing the sign-time distribution. We believe that this distribution will become an important new tool in other persistence-type problems. The tails of the distribution contain standard persistence information, whilst the body of $P$ gives important information regarding the mixing efficiency of the problem at hand. If $P$ is concave (convex), than the mean value is the least (most) likely. Such information is important when ergodic properties of a system are under investigation. The fact that the curvature of $P$ for the simple diffusion equation 'flips' at $d=d_{c} \simeq 36$ indicates that the dynamics in the phase space of few-body systems may be extremely sensitive to exactly how many degrees of freedom are considered.

The authors are grateful to A. Bray, E. Ben-Naim, C. Godrèche and R. Zia for interesting discussions and also thank C. Godrèche for bringing Ref. 8 to their attention prior to publication. T.J.N. and Z.T. acknowledge financial support from the Materials Research Division of the National Science Foundation. Z.T. also acknowledges support from the Hungarian Science Foundation, T17493 and T19483.

\section{APPENDIX A:}

In this appendix we sketch a brief proof of the assertion that $P$ satisfies the exact scaling form $P[\tau, t] d \tau=$ $f(\tau / t)(d \tau / t)$ for all $t$. First, we note that the sign-time distribution can be written as $P[\tau, t]=\left\langle\delta\left(\tau-\tau_{\psi}\right)\right\rangle_{R}$, where $\tau_{\psi}$ is the implicit function of $\psi$ given in (5). Thus,

$$
P[\tau, t]=\int_{-\infty}^{\infty} \frac{d \omega}{2 \pi} e^{i \omega \tau}\left\langle\exp \left(-i \omega \tau_{\psi}\right)\right\rangle_{R}
$$

The average on the rhs of the above expression may be re-expressed as a power series in terms of $\omega^{n}\left\langle\tau_{\psi}^{n}\right\rangle_{R}$. The $n^{\text {th }}$ moment is an $n$-fold integral over the average of $n$ step-functions. Each step-function may be represented by an integral, yielding (with an implicit limit of $\epsilon_{k} \rightarrow 0$ )

$$
\left\langle\tau_{\psi}^{n}\right\rangle=\frac{1}{(2 \pi)^{n}} \prod_{j=1}^{n} \int_{0}^{t} d t_{k} \int_{-\infty}^{\infty} \frac{d \sigma_{k}}{\left(\epsilon_{k}+i \sigma_{k}\right)}\left\langle e^{i \sum_{l} \sigma_{l} \phi\left(\mathbf{0}, t_{l}\right)}\right\rangle_{R} .
$$

The average is easily performed over the gaussian distribution $R[\psi]$. On rescaling the integration variables we find

$$
\left\langle\tau_{\psi}^{n}\right\rangle=\left(\frac{t}{2 \pi}\right)^{n} \prod_{k=1}^{n} \int_{0}^{1} d a_{k} \int_{-\infty}^{\infty} \frac{d \sigma_{k}^{\prime}}{\left(\epsilon_{k}^{\prime}+i \sigma_{k}^{\prime}\right)}\left[e^{-\sum_{l, m} \sigma_{l}^{\prime} M_{l, m} \sigma_{m}^{\prime}}\right]
$$

where $M_{l, m}=\left[2 a_{l}^{1 / 2} a_{m}^{1 / 2} /\left(a_{l}+a_{m}\right)\right]^{d / 2}$. We see that the $n^{\text {th }}$ moment scales exactly as $t^{n}$, which enables us to combine $\omega$ and $t$ as a simple product. Returning to (A1), we may scale $\omega$ by $t$ to obtain the desired result.

[1] S. N. Majumdar et al., Phys. Rev. Lett., 772867 (1996).

[2] B. Derrida et al., J. Phys. A, 27 L357 (1994).

[3] B. Derrida et al., Phys. Rev. Lett., 75751 (1995).

[4] J. Krug et al., Phys. Rev. E 56 (1997) 2702

[5] B. P. Lee et al., Phys. Rev. Lett., 794842 (1997).

[6] M. Howard and C. Godrèche, cond-mat/9711148 (1997).

[7] J. M. Burgers, The Non-linear Diffusion Equation (Reidel, Boston, 1974). 
[8] I. Dornic and C. Godrèche, J. Phys. A (to appear) (1998).

[9] H. Jeffreys and B. Jeffreys, Methods of Mathematical Physics 3rd Edition (Cambridge University Press, Cambridge, 1980).

[10] Handbook of Mathematical Functions 10th Edition, M. Abramowitz and I. A. Stegun Eds. (Dover, NY, 1972).

[11] L. Schläfli, Quart. J. Pure and Appl. Math., 2 269-301 (1858); ibid 3 54-68, 97-108 (1860); H. S. M. Coxeter, Regular Polytopes 2nd Edition, (MacMillan, New York, 1963).

[12] Z. Toroczkai and T. J. Newman, in preparation. 